\title{
Phenylthiophene-dipicolinic acid-based emitters with strong solution blue and solid state green emission
}

\author{
Ana de Bettencourt-Dias*, Andrei Poloukhtine.
}

$\underline{\text { Table of Contents for the Supplementary Material }}$

Crystal structure determinations

Page S2

Calculations

Page S2

Table S1. Crystallographic details for compound 1.

Page S3

Table S2. Bond lengths $[\AA]$ and angles $\left[{ }^{\circ}\right]$ for $\mathbf{1}$.

Page S4

Figure S1. ${ }^{1} \mathrm{H}-\mathrm{NMR}$ spectrum of compound a in $\mathrm{CDCl}_{3}$.

Page S7

Figure S2. ${ }^{1} \mathrm{H}-\mathrm{NMR}$ spectrum of compound $\mathbf{b}$ in $\mathrm{CDCl}_{3}$.

Page S8

Figure S3. ${ }^{1} \mathrm{H}-\mathrm{NMR}$ spectrum of compound $\mathbf{d}$ in $\mathrm{CDCl}_{3}$.

Page S9

Figure S4. ${ }^{1} \mathrm{H}-\mathrm{NMR}$ spectrum of compound $\mathbf{e}$ in $\mathrm{CDCl}_{3}$.

Page S10

Figure S5. ${ }^{1} \mathrm{H}-\mathrm{NMR}$ of compound 1 in $\mathrm{CDCl}_{3}$.

Page S11

Figure S6. ${ }^{13} \mathrm{C}-\mathrm{NMR}$ of compound 1 in $\mathrm{CDCl}_{3}$.

Page S12

Figure S7. ${ }^{1} \mathrm{H}-\mathrm{NMR}$ of compound 2 in $\mathrm{CDCl}_{3}$.

Page S13

Figure S8. ${ }^{13} \mathrm{C}-\mathrm{NMR}$ of compound 2 in $\mathrm{CDCl}_{3}$.

Page S14

Figure S9. ${ }^{1} \mathrm{H}-\mathrm{NMR}$ of compound 3 in $\mathrm{CDCl}_{3}$.

Page S15

Figure S10. ${ }^{13} \mathrm{C}-\mathrm{NMR}$ of compound 3 in $\mathrm{CDCl}_{3}$.

Page S16

Bibliography

Page S16 


\section{Crystal structure determinations}

Crystal data, data collection and refinement details for compound $\mathbf{1}$ are given in the Supplmentary information in Table S1 and selected bond lengths are given in Table S2. A suitable crystal was mounted on a glass fiber and placed in the low-temperature nitrogen stream. Data were collected on a Bruker SMART CCD area detector diffractometer equipped with a lowtemperature device, ${ }^{5}$ using graphite-monochromated Mo-K $\alpha$ radiation $(\lambda=0.71073 \AA)$. Data were measured using omega scans of $0.3^{\circ}$ per frame and a full sphere of data was collected, for a total of 1850 frames. The first 50 frames were recollected at the end of data collection to monitor for decay. Multi-scan absorption corrections were applied using SADABS. ${ }^{6}$. Cell parameters were retrieved using $\mathrm{SMART}^{7}$ software and refined using SAINTPlus ${ }^{8}$ on all observed reflections. Data reduction and correction for Lp and decay were performed using the SAINTPlus ${ }^{8}$ software. The structures were solved by direct methods and refined by least square methods on $F^{2}$ using the SHELXTL ${ }^{9}$ program package. All non-hydrogen atoms were refined anisotropically. The majority of the hydrogen atoms were added geometrically and their parameters constrained to the parent site. The X-ray crystallographic information file CCDC 296287 can be obtained free of charge via www.ccdc.cam.ac.uk/conts/retrieving.html (or from the CCDC, 12 Union Road, Cambridge CB2 1EZ, UK; fax: +44 1223 336033; e-mail: deposit@ccdc.cam.ac.uk). Powder data were collected on a Bruker D8 Advance (Cu-Ko radiation, $5-60^{\circ}, 0.01^{\circ}$ step, $0.5^{\circ} \mathrm{s} /$ step).

\section{Calculations}

DFT calculations were performed using the B3LYP method with the 6-31G(d) basis set. The geometries of the three compounds were optimized with $\mathbf{1}$ and $\mathbf{2}$ constrained to the $C_{i}$ point group 
and 3 without constraints. The initial geometries were derived from the conformation of $\mathbf{1}$ obtained by single crystal X-ray diffraction. Gaussian 03 was utilized for all calculations. ${ }^{10}$

Table S1. Crystallographic details for compound 1.

\begin{tabular}{ll}
\hline Compound & $\mathbf{1}$ \\
\hline CCDC number & 296287 \\
\hline Formula & $\mathrm{C}_{44} \mathrm{H}_{52} \mathrm{~N}_{6} \mathrm{O}_{4} \mathrm{~S}_{2}$ \\
\hline $\mathrm{M} / \mathrm{g} \mathrm{mol}^{-1}$ & 793.04 \\
\hline Crystal system & Monoclinic \\
\hline Space group & $P 2_{1} / \mathrm{c}$ \\
\hline$a / \AA$ & $15.224(3)$ \\
\hline$b / \AA$ & $8.9383(18)$ \\
\hline$c / \AA$ & $17.234(3)$ \\
\hline$\beta l^{0}$ & $114.36(3)$ \\
\hline$V / \AA^{3}$ & $2136.4(7)$ \\
\hline$T / \mathrm{K}$ & $100(2)$ \\
\hline$Z$ & 2 \\
\hline$D_{c} / \mathrm{g} \mathrm{cm}{ }^{-3}$ & 1.233 \\
\hline$\mu($ Mo-K $\alpha) / m m^{-1}$ & 0.173 \\
\hline Independent reflections, $R_{\text {int }}\left[F_{0} \geq 4 \sigma\left(F_{0}\right)\right]$ & $5313,0.0800$ \\
\hline Reflections collected & 21847 \\
\hline$R_{1}, w R_{2}(I>2 \sigma(I))$ & $0.0575,0.1231$ \\
\hline$R_{1}, w R_{2}(a l l ~ d a t a)$ & $0.1055,0.1436$ \\
\hline Largest diff. peak and hole/ $\AA^{-3}$ & $0.969,-0.398$ \\
\hline & \\
\hline
\end{tabular}


Table S2. Bond lengths $[\AA]$ and angles $\left[^{\circ}\right]$ for $\mathbf{1}$.

\begin{tabular}{|c|c|c|c|}
\hline$C(1)-C(2)$ & $1.357(3)$ & $\mathrm{C}(12)-\mathrm{H}(12)$ & 0.9500 \\
\hline C(1)-C(10) & $1.466(3)$ & $\mathrm{C}(13)-\mathrm{O}(1)$ & $1.231(3)$ \\
\hline $\mathrm{C}(1)-\mathrm{S}(1)$ & $1.727(2)$ & $\mathrm{C}(13)-\mathrm{N}(2)$ & $1.348(3)$ \\
\hline $\mathrm{C}(2)-\mathrm{C}(3)$ & $1.402(3)$ & $\mathrm{C}(14)-\mathrm{O}(2)$ & $1.233(3)$ \\
\hline $\mathrm{C}(2)-\mathrm{H}(2)$ & 0.9500 & $\mathrm{C}(14)-\mathrm{N}(3)$ & $1.349(3)$ \\
\hline $\mathrm{C}(3)-\mathrm{C}(4)$ & $1.362(3)$ & $\mathrm{C}(15)-\mathrm{N}(2)$ & $1.470(3)$ \\
\hline C(3)-H(3) & 0.9500 & $\mathrm{C}(15)-\mathrm{C}(16)$ & $1.527(4)$ \\
\hline$C(4)-C(7)$ & $1.459(3)$ & $\mathrm{C}(15)-\mathrm{H}(15 \mathrm{~A})$ & 0.9900 \\
\hline$C(4)-S(1)$ & $1.733(2)$ & $\mathrm{C}(15)-\mathrm{H}(15 \mathrm{~B})$ & 0.9900 \\
\hline $\mathrm{C}(5)-\mathrm{N}(1)$ & $1.344(3)$ & $\mathrm{C}(16)-\mathrm{H}(16 \mathrm{~A})$ & 0.9800 \\
\hline$C(5)-C(6)$ & 1.383(3) & $\mathrm{C}(16)-\mathrm{H}(16 \mathrm{~B})$ & 0.9800 \\
\hline C(5)-C(13) & $1.512(3)$ & $\mathrm{C}(16)-\mathrm{H}(16 \mathrm{C})$ & 0.9800 \\
\hline$C(6)-C(7)$ & $1.396(3)$ & $\mathrm{C}(17)-\mathrm{N}(2)$ & 1.461(3) \\
\hline $\mathrm{C}(6)-\mathrm{H}(6)$ & 0.9500 & $\mathrm{C}(17)-\mathrm{C}(18)$ & $1.515(4)$ \\
\hline$C(7)-C(8)$ & $1.400(3)$ & $\mathrm{C}(17)-\mathrm{H}(17 \mathrm{~A})$ & 0.9900 \\
\hline $\mathrm{C}(8)-\mathrm{C}(9)$ & 1.383(3) & $\mathrm{C}(17)-\mathrm{H}(17 \mathrm{~B})$ & 0.9900 \\
\hline $\mathrm{C}(8)-\mathrm{H}(8)$ & 0.9500 & $\mathrm{C}(18)-\mathrm{H}(18 \mathrm{~A})$ & 0.9800 \\
\hline $\mathrm{C}(9)-\mathrm{N}(1)$ & $1.341(3)$ & $\mathrm{C}(18)-\mathrm{H}(18 \mathrm{~B})$ & 0.9800 \\
\hline C(9)-C(14) & $1.507(3)$ & $\mathrm{C}(18)-\mathrm{H}(18 \mathrm{C})$ & 0.9800 \\
\hline $\mathrm{C}(10)-\mathrm{C}(11)$ & 1.399(3) & C(19)-N(3) & $1.462(3)$ \\
\hline $\mathrm{C}(10)-\mathrm{C}(12)$ & $1.400(3)$ & $C(19)-C(20)$ & $1.515(4)$ \\
\hline $\mathrm{C}(11)-\mathrm{C}(12) \# 1$ & 1.383(3) & $\mathrm{C}(19)-\mathrm{H}(19 \mathrm{~A})$ & 0.9900 \\
\hline $\mathrm{C}(11)-\mathrm{H}(11)$ & 0.9500 & C(19)-H(19B) & 0.9900 \\
\hline $\mathrm{C}(12)-\mathrm{C}(11) \# 1$ & $1.383(3)$ & $\mathrm{C}(20)-\mathrm{H}(20 \mathrm{~A})$ & 0.9800 \\
\hline
\end{tabular}




\begin{tabular}{|c|c|c|c|}
\hline $\mathrm{C}(20)-\mathrm{H}(20 \mathrm{~B})$ & 0.9800 & $\mathrm{C}(7)-\mathrm{C}(6)-\mathrm{H}(6)$ & 120.1 \\
\hline C(20)-H(20C) & 0.9800 & $\mathrm{C}(6)-\mathrm{C}(7)-\mathrm{C}(8)$ & $116.8(2)$ \\
\hline $\mathrm{C}(21)-\mathrm{N}(3)$ & $1.469(3)$ & $C(6)-C(7)-C(4)$ & $121.0(2)$ \\
\hline $\mathrm{C}(21)-\mathrm{C}(22)$ & $1.513(4)$ & $C(8)-C(7)-C(4)$ & $122.1(2)$ \\
\hline $\mathrm{C}(21)-\mathrm{H}(21 \mathrm{~A})$ & 0.9900 & $\mathrm{C}(9)-\mathrm{C}(8)-\mathrm{C}(7)$ & 119.6(2) \\
\hline $\mathrm{C}(21)-\mathrm{H}(21 \mathrm{~B})$ & 0.9900 & $\mathrm{C}(9)-\mathrm{C}(8)-\mathrm{H}(8)$ & 120.2 \\
\hline $\mathrm{C}(22)-\mathrm{H}(22 \mathrm{~A})$ & 0.9800 & $\mathrm{C}(7)-\mathrm{C}(8)-\mathrm{H}(8)$ & 120.2 \\
\hline $\mathrm{C}(22)-\mathrm{H}(22 \mathrm{~B})$ & 0.9800 & N(1)-C(9)-C(8) & 123.3(2) \\
\hline \multirow[t]{2}{*}{$\mathrm{C}(22)-\mathrm{H}(22 \mathrm{C})$} & 0.9800 & $\mathrm{~N}(1)-\mathrm{C}(9)-\mathrm{C}(14)$ & 116.91(19) \\
\hline & & $\mathrm{C}(8)-\mathrm{C}(9)-\mathrm{C}(14)$ & 119.2(2) \\
\hline$C(2)-C(1)-C(10)$ & $127.7(2)$ & $\mathrm{C}(11)-\mathrm{C}(10)-\mathrm{C}(12)$ & $119.0(2)$ \\
\hline $\mathrm{C}(2)-\mathrm{C}(1)-\mathrm{S}(1)$ & $110.42(18)$ & $\mathrm{C}(11)-\mathrm{C}(10)-\mathrm{C}(1)$ & 119.6(2) \\
\hline $\mathrm{C}(10)-\mathrm{C}(1)-\mathrm{S}(1)$ & 121.92(18) & $\mathrm{C}(12)-\mathrm{C}(10)-\mathrm{C}(1)$ & $121.4(2)$ \\
\hline $\mathrm{C}(1)-\mathrm{C}(2)-\mathrm{C}(3)$ & 113.6(2) & $\mathrm{C}(12) \# 1-\mathrm{C}(11)-\mathrm{C}(10)$ & $120.5(2)$ \\
\hline $\mathrm{C}(1)-\mathrm{C}(2)-\mathrm{H}(2)$ & 123.2 & $\mathrm{C}(12) \# 1-\mathrm{C}(11)-\mathrm{H}(11)$ & 119.8 \\
\hline $\mathrm{C}(3)-\mathrm{C}(2)-\mathrm{H}(2)$ & 123.2 & $\mathrm{C}(10)-\mathrm{C}(11)-\mathrm{H}(11)$ & 119.8 \\
\hline $\mathrm{C}(4)-\mathrm{C}(3)-\mathrm{C}(2)$ & 113.7(2) & $\mathrm{C}(11) \# 1-\mathrm{C}(12)-\mathrm{C}(10)$ & $120.5(2)$ \\
\hline C(4)-C(3)-H(3) & 123.1 & $\mathrm{C}(11) \# 1-\mathrm{C}(12)-\mathrm{H}(12)$ & 119.7 \\
\hline $\mathrm{C}(2)-\mathrm{C}(3)-\mathrm{H}(3)$ & 123.1 & $\mathrm{C}(10)-\mathrm{C}(12)-\mathrm{H}(12)$ & 119.7 \\
\hline$C(3)-C(4)-C(7)$ & $128.3(2)$ & $\mathrm{O}(1)-\mathrm{C}(13)-\mathrm{N}(2)$ & $123.2(2)$ \\
\hline $\mathrm{C}(3)-\mathrm{C}(4)-\mathrm{S}(1)$ & 109.96(18) & $\mathrm{O}(1)-\mathrm{C}(13)-\mathrm{C}(5)$ & $117.5(2)$ \\
\hline $\mathrm{C}(7)-\mathrm{C}(4)-\mathrm{S}(1)$ & $121.70(17)$ & $\mathrm{N}(2)-\mathrm{C}(13)-\mathrm{C}(5)$ & 119.3(2) \\
\hline N(1)-C(5)-C(6) & 123.2(2) & $\mathrm{O}(2)-\mathrm{C}(14)-\mathrm{N}(3)$ & $122.9(2)$ \\
\hline $\mathrm{N}(1)-\mathrm{C}(5)-\mathrm{C}(13)$ & 118.17(19) & $\mathrm{O}(2)-\mathrm{C}(14)-\mathrm{C}(9)$ & $118.0(2)$ \\
\hline$C(6)-C(5)-C(13)$ & 118.5(2) & $\mathrm{N}(3)-\mathrm{C}(14)-\mathrm{C}(9)$ & $118.9(2)$ \\
\hline$C(5)-C(6)-C(7)$ & 119.7(2) & $\mathrm{N}(2)-\mathrm{C}(15)-\mathrm{C}(16)$ & $112.6(2)$ \\
\hline $\mathrm{C}(5)-\mathrm{C}(6)-\mathrm{H}(6)$ & 120.1 & $\mathrm{~N}(2)-\mathrm{C}(15)-\mathrm{H}(15 \mathrm{~A})$ & 109.1 \\
\hline
\end{tabular}




\begin{tabular}{|c|c|c|c|}
\hline C(16)-C(15)-H(15A) & 109.1 & H(19A)-C(19)-H(19B) & 107.9 \\
\hline $\mathrm{N}(2)-\mathrm{C}(15)-\mathrm{H}(15 \mathrm{~B})$ & 109.1 & $\mathrm{C}(19)-\mathrm{C}(20)-\mathrm{H}(20 \mathrm{~A})$ & 109.5 \\
\hline C(16)-C(15)-H(15B) & 109.1 & $\mathrm{C}(19)-\mathrm{C}(20)-\mathrm{H}(20 \mathrm{~B})$ & 109.5 \\
\hline $\mathrm{H}(15 \mathrm{~A})-\mathrm{C}(15)-\mathrm{H}(15 \mathrm{~B})$ & 107.8 & $H(20 A)-C(20)-H(20 B)$ & 109.5 \\
\hline $\mathrm{C}(15)-\mathrm{C}(16)-\mathrm{H}(16 \mathrm{~A})$ & 109.5 & $\mathrm{C}(19)-\mathrm{C}(20)-\mathrm{H}(20 \mathrm{C})$ & 109.5 \\
\hline $\mathrm{C}(15)-\mathrm{C}(16)-\mathrm{H}(16 \mathrm{~B})$ & 109.5 & $H(20 A)-C(20)-H(20 C)$ & 109.5 \\
\hline $\mathrm{H}(16 \mathrm{~A})-\mathrm{C}(16)-\mathrm{H}(16 \mathrm{~B})$ & 109.5 & $\mathrm{H}(20 \mathrm{~B})-\mathrm{C}(20)-\mathrm{H}(20 \mathrm{C})$ & 109.5 \\
\hline C(15)-C(16)-H(16C) & 109.5 & N(3)-C(21)-C(22) & $109.9(2)$ \\
\hline $\mathrm{H}(16 \mathrm{~A})-\mathrm{C}(16)-\mathrm{H}(16 \mathrm{C})$ & 109.5 & $\mathrm{~N}(3)-\mathrm{C}(21)-\mathrm{H}(21 \mathrm{~A})$ & 109.7 \\
\hline H(16B)-C(16)-H(16C) & 109.5 & $C(22)-C(21)-H(21 A)$ & 109.7 \\
\hline $\mathrm{N}(2)-\mathrm{C}(17)-\mathrm{C}(18)$ & $113.3(2)$ & $\mathrm{N}(3)-\mathrm{C}(21)-\mathrm{H}(21 \mathrm{~B})$ & 109.7 \\
\hline N(2)-C(17)-H(17A) & 108.9 & $\mathrm{C}(22)-\mathrm{C}(21)-\mathrm{H}(21 \mathrm{~B})$ & 109.7 \\
\hline $\mathrm{C}(18)-\mathrm{C}(17)-\mathrm{H}(17 \mathrm{~A})$ & 108.9 & $\mathrm{H}(21 \mathrm{~A})-\mathrm{C}(21)-\mathrm{H}(21 \mathrm{~B})$ & 108.2 \\
\hline N(2)-C(17)-H(17B) & 108.9 & $\mathrm{C}(21)-\mathrm{C}(22)-\mathrm{H}(22 \mathrm{~A})$ & 109.5 \\
\hline C(18)-C(17)-H(17B) & 108.9 & $\mathrm{C}(21)-\mathrm{C}(22)-\mathrm{H}(22 \mathrm{~B})$ & 109.5 \\
\hline $\mathrm{H}(17 \mathrm{~A})-\mathrm{C}(17)-\mathrm{H}(17 \mathrm{~B})$ & 107.7 & $\mathrm{H}(22 \mathrm{~A})-\mathrm{C}(22)-\mathrm{H}(22 \mathrm{~B})$ & 109.5 \\
\hline C(17)-C(18)-H(18A) & 109.5 & $\mathrm{C}(21)-\mathrm{C}(22)-\mathrm{H}(22 \mathrm{C})$ & 109.5 \\
\hline C(17)-C(18)-H(18B) & 109.5 & $\mathrm{H}(22 \mathrm{~A})-\mathrm{C}(22)-\mathrm{H}(22 \mathrm{C})$ & 109.5 \\
\hline $\mathrm{H}(18 \mathrm{~A})-\mathrm{C}(18)-\mathrm{H}(18 \mathrm{~B})$ & 109.5 & $\mathrm{H}(22 \mathrm{~B})-\mathrm{C}(22)-\mathrm{H}(22 \mathrm{C})$ & 109.5 \\
\hline C(17)-C(18)-H(18C) & 109.5 & $\mathrm{C}(9)-\mathrm{N}(1)-\mathrm{C}(5)$ & 117.19(19) \\
\hline H(18A)-C(18)-H(18C) & 109.5 & $\mathrm{C}(13)-\mathrm{N}(2)-\mathrm{C}(17)$ & $125.5(2)$ \\
\hline H(18B)-C(18)-H(18C) & 109.5 & $\mathrm{C}(13)-\mathrm{N}(2)-\mathrm{C}(15)$ & $117.9(2)$ \\
\hline N(3)-C(19)-C(20) & $111.7(2)$ & $\mathrm{C}(17)-\mathrm{N}(2)-\mathrm{C}(15)$ & $116.2(2)$ \\
\hline N(3)-C(19)-H(19A) & 109.3 & $\mathrm{C}(14)-\mathrm{N}(3)-\mathrm{C}(19)$ & $124.8(2)$ \\
\hline C(20)-C(19)-H(19A) & 109.3 & $\mathrm{C}(14)-\mathrm{N}(3)-\mathrm{C}(21)$ & $118.1(2)$ \\
\hline N(3)-C(19)-H(19B) & 109.3 & $\mathrm{C}(19)-\mathrm{N}(3)-\mathrm{C}(21)$ & $116.8(2)$ \\
\hline C(20)-C(19)-H(19B) & 109.3 & $\mathrm{C}(1)-\mathrm{S}(1)-\mathrm{C}(4)$ & $92.26(11)$ \\
\hline
\end{tabular}


Symmetry transformations used to generate equivalent atoms: \#1 -x+1,-y+2,-z+2

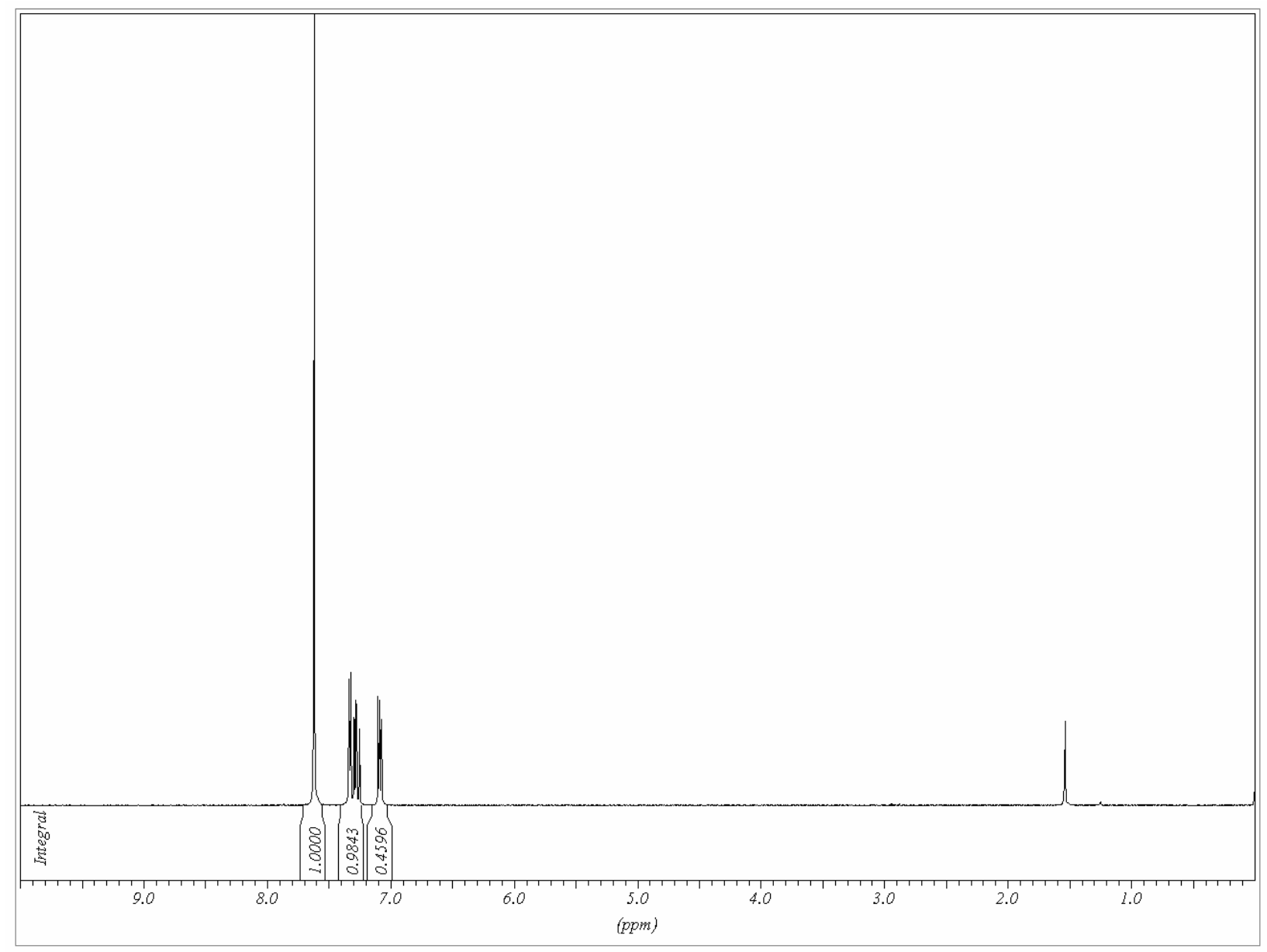

Figure S1. ${ }^{1} \mathrm{H}-\mathrm{NMR}$ spectrum of compound a in $\mathrm{CDCl}_{3}$. Peak at $1.535 \mathrm{ppm}$ is water. 


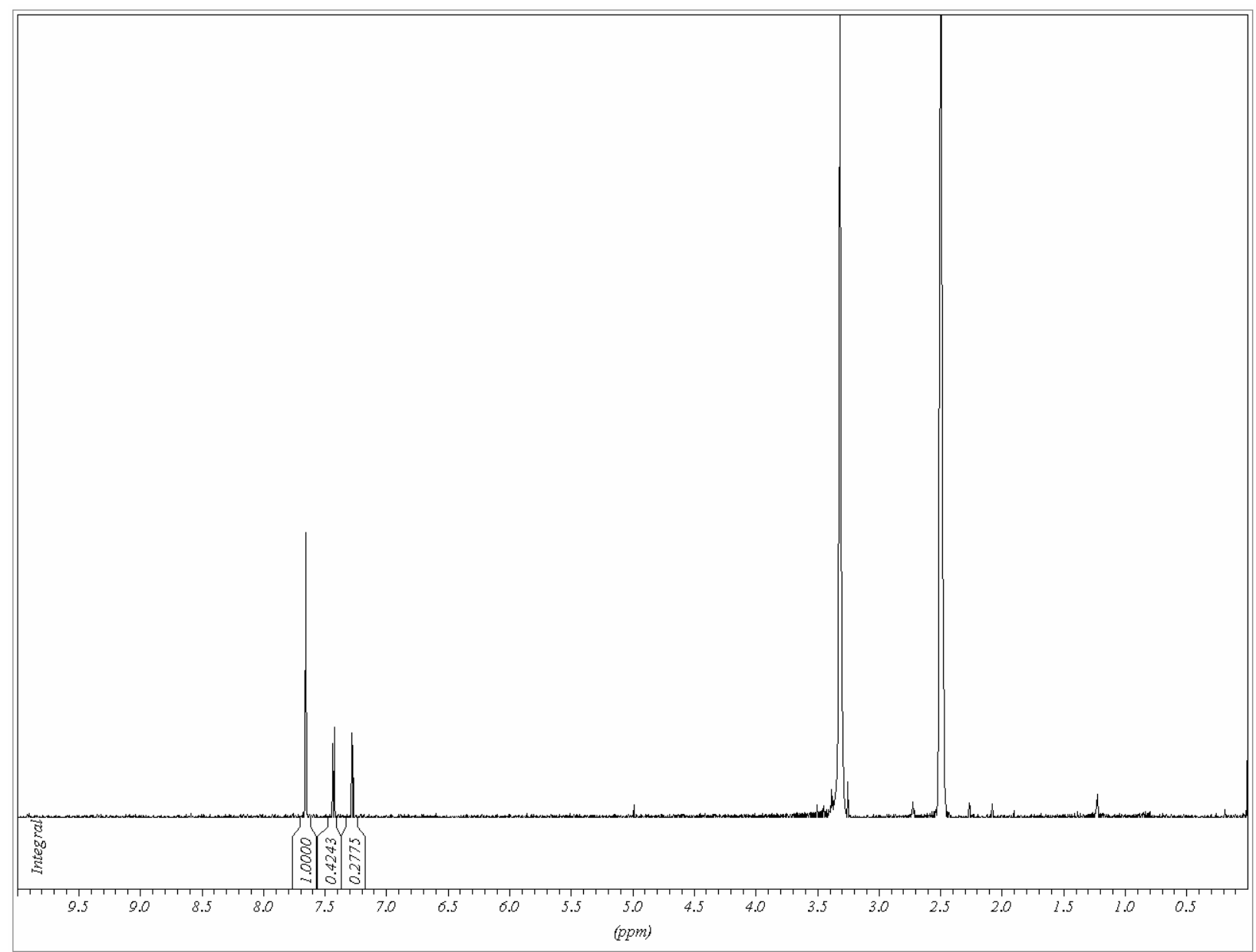

Figure S2. ${ }^{1} \mathrm{H}-\mathrm{NMR}$ spectrum of compound a in $d^{6}$-DMSO. Peak at $3.248 \mathrm{ppm}$ is water. 


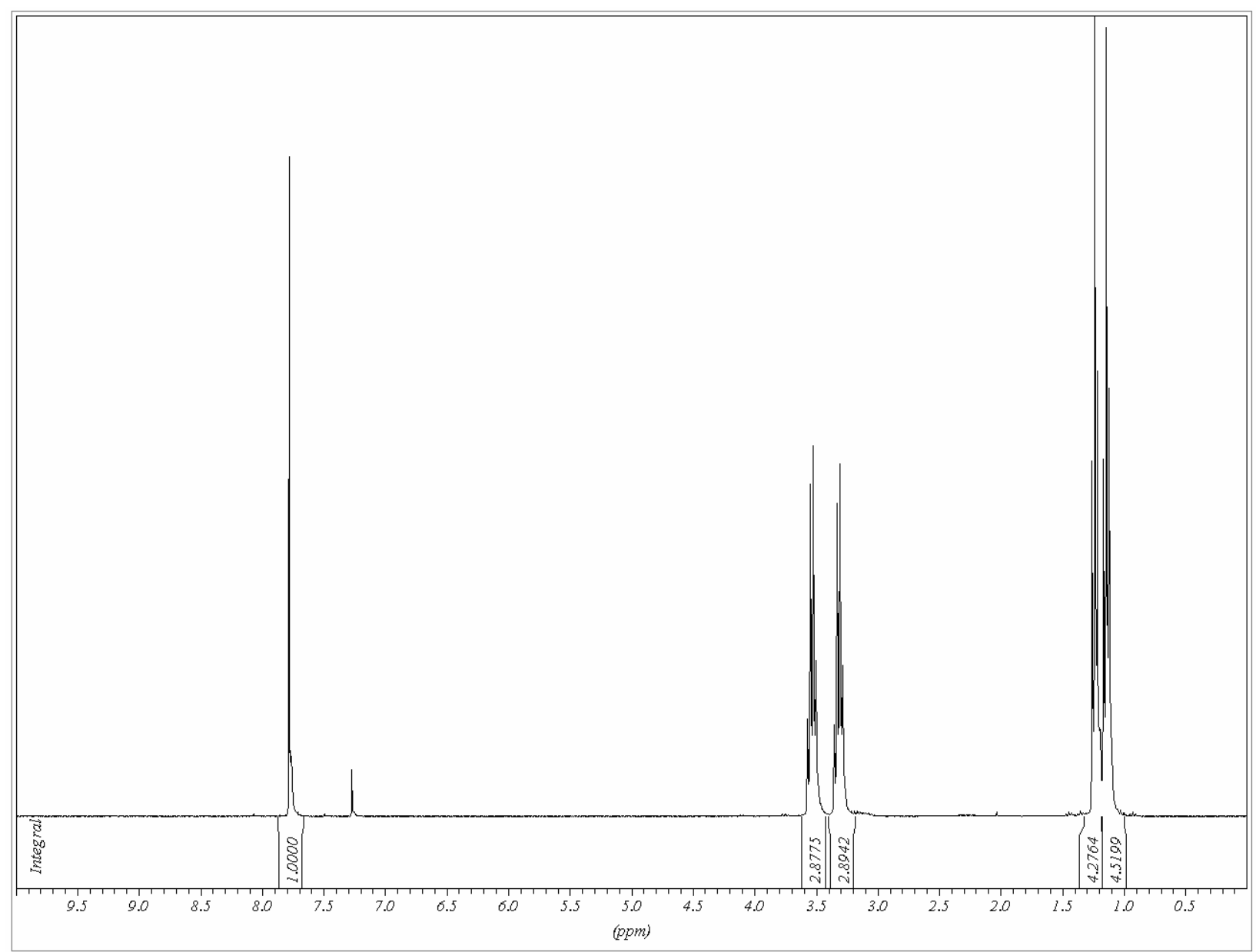

Figure S3. ${ }^{1} \mathrm{H}-\mathrm{NMR}$ spectrum of compound $\mathbf{d}$ in $\mathrm{CDCl}_{3}$. 


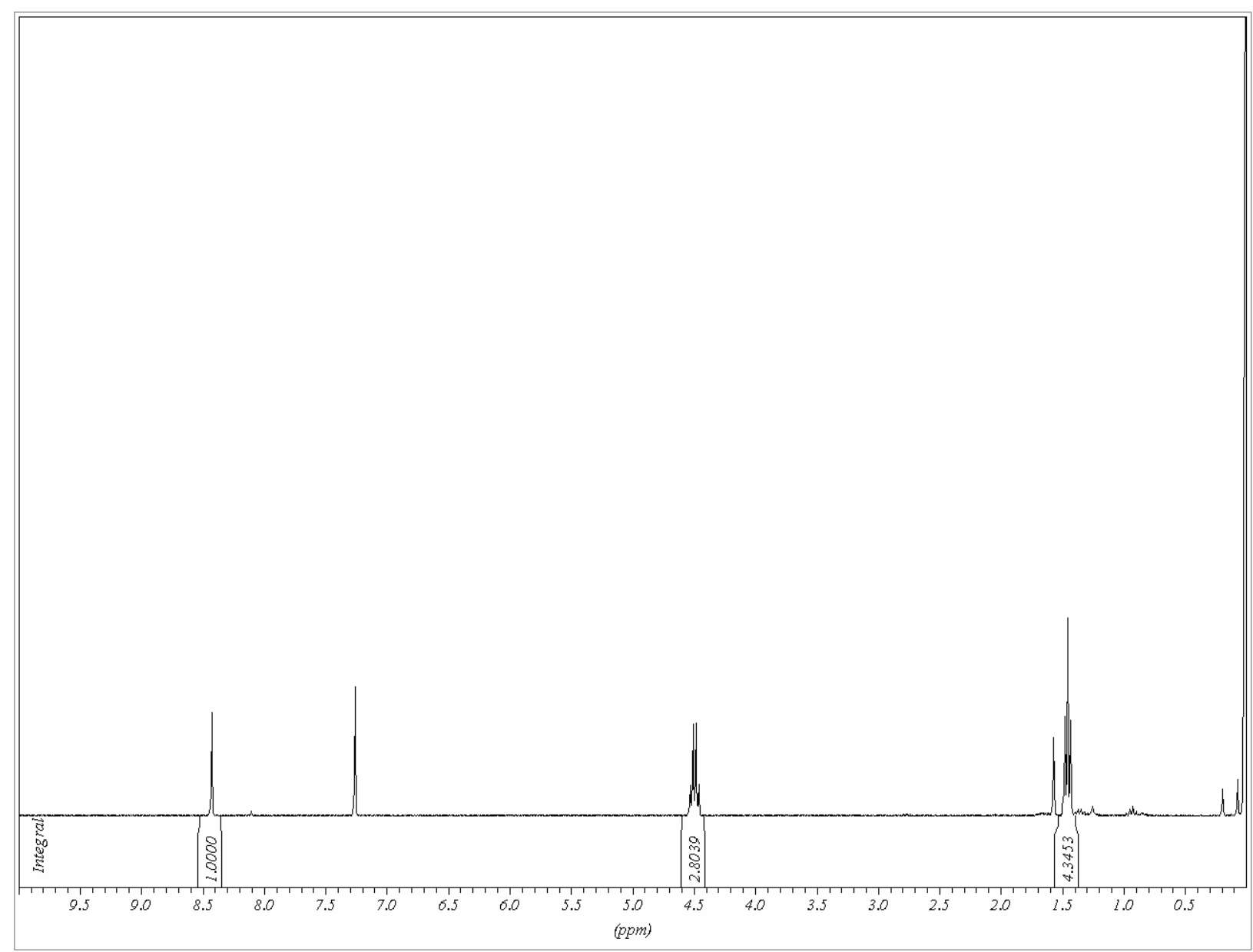

Figure S4. ${ }^{1} \mathrm{H}-\mathrm{NMR}$ spectrum of compound $\mathbf{e}$ in $\mathrm{CDCl}_{3}$. 


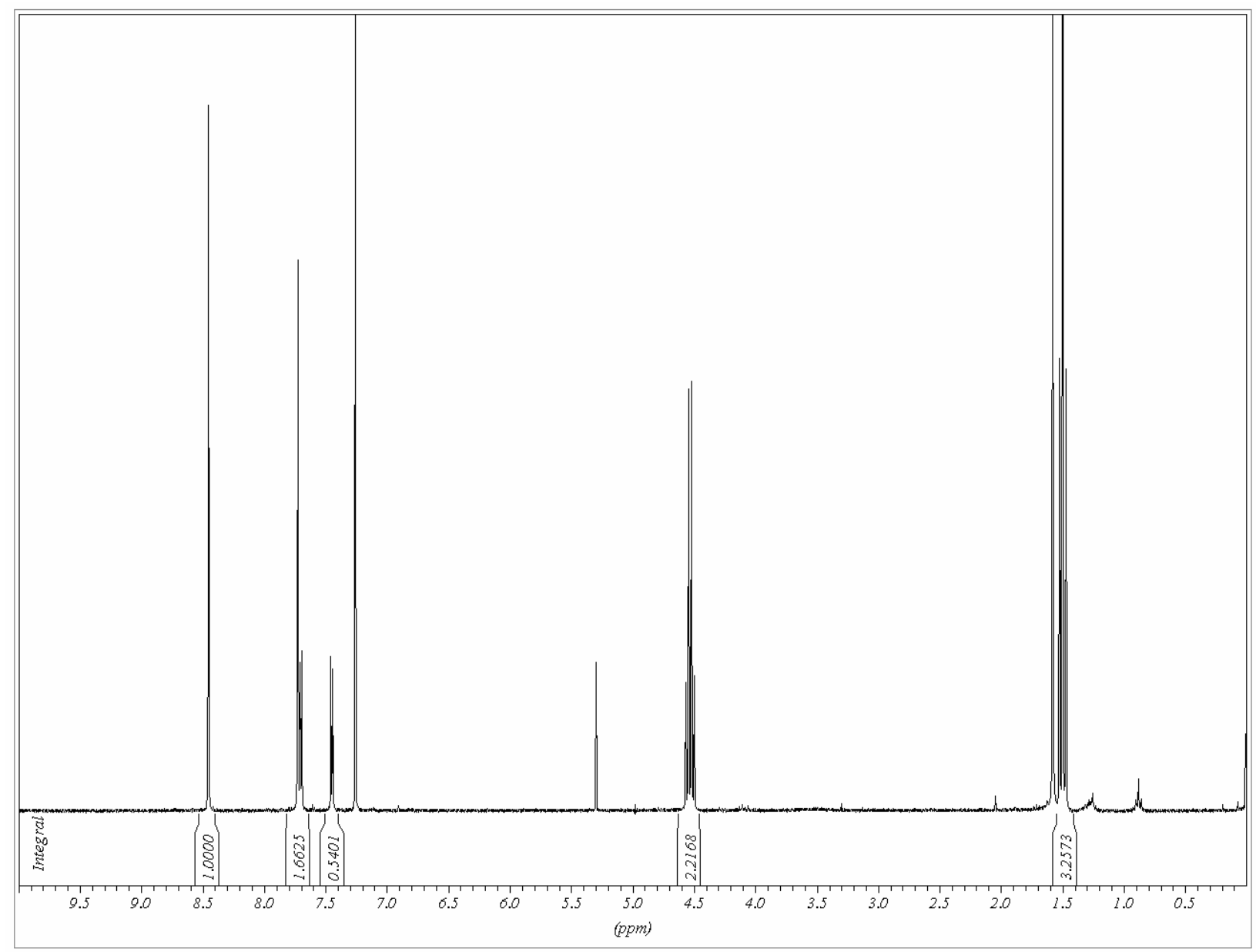

Figure S5. ${ }^{1} \mathrm{H}-\mathrm{NMR}$ of compound $\mathbf{1}$ in $\mathrm{CDCl}_{3}$. Peaks not integrated correspond to water at $1.579 \mathrm{ppm}$ and $5.2995 \mathrm{ppm}$ for $\mathrm{CH}_{2} \mathrm{Cl}_{2}$. 


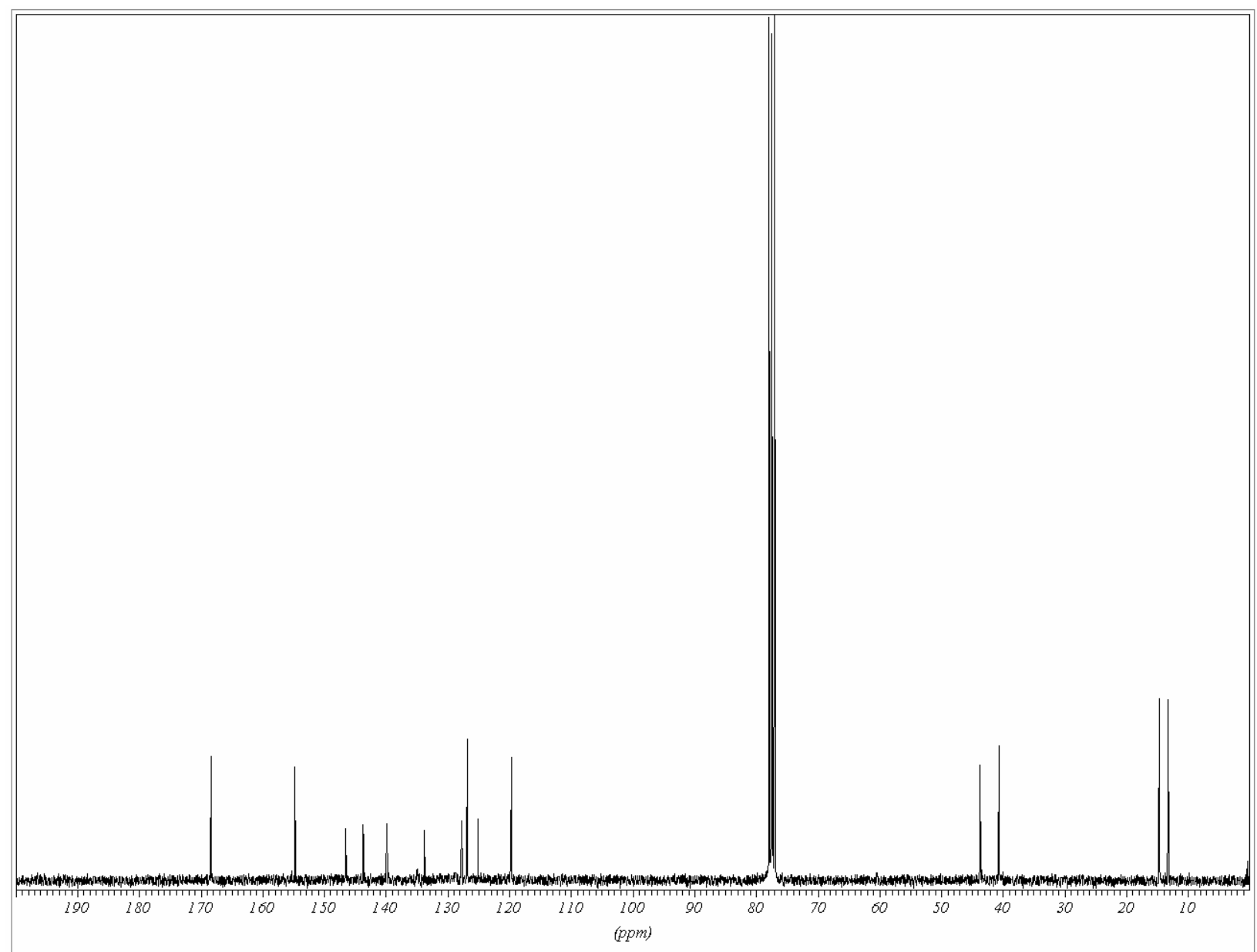

Figure S6. ${ }^{13} \mathrm{C}-\mathrm{NMR}$ of compound 1 in $\mathrm{CDCl}_{3}$. 


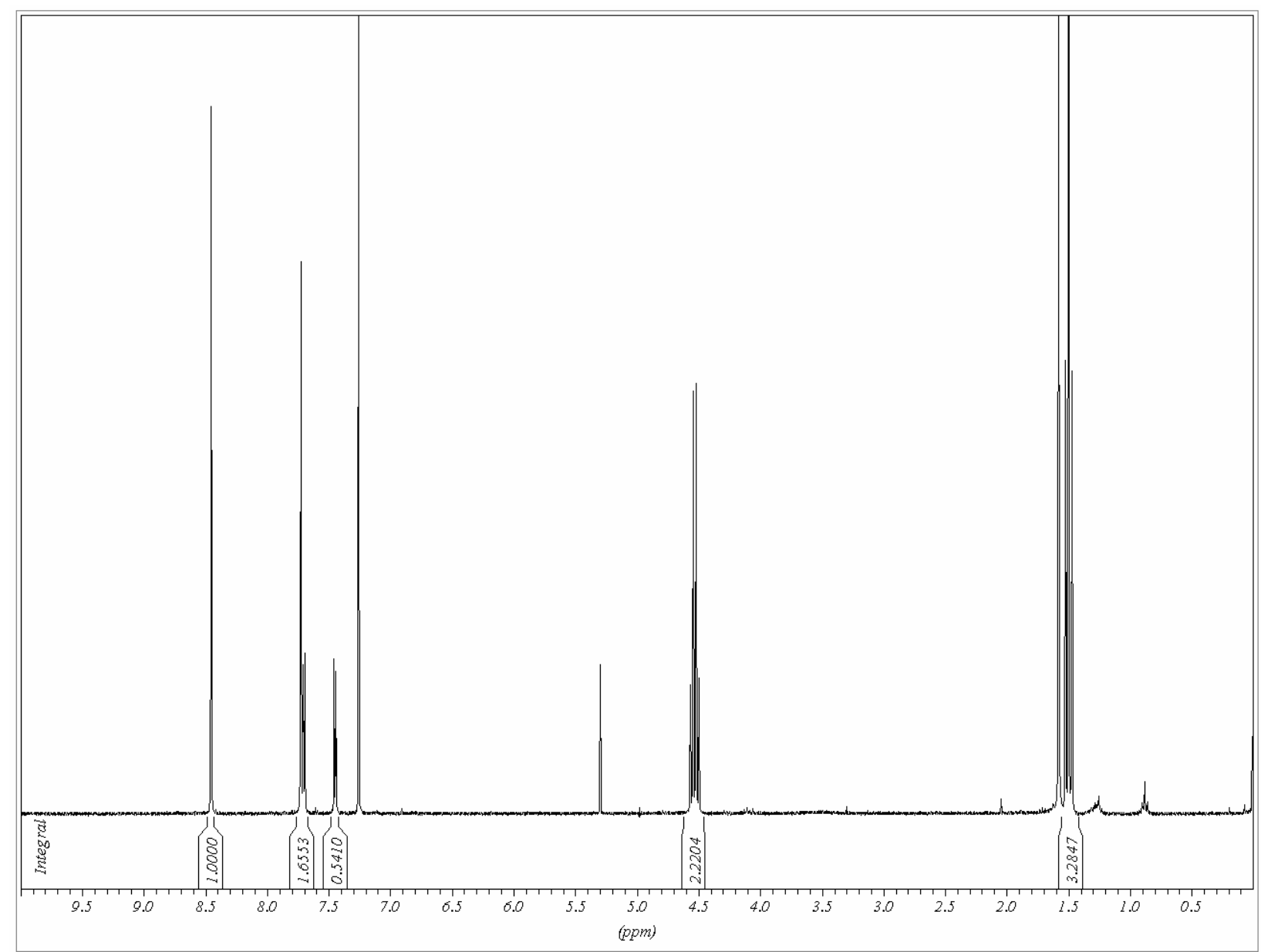

Figure S7. ${ }^{1} \mathrm{H}-\mathrm{NMR}$ of compound 2 in $\mathrm{CDCl}_{3}$. Peaks not integrated correspond to water at $1.579 \mathrm{ppm}$ and $5.2995 \mathrm{ppm}$ for $\mathrm{CH}_{2} \mathrm{Cl}_{2}$. 


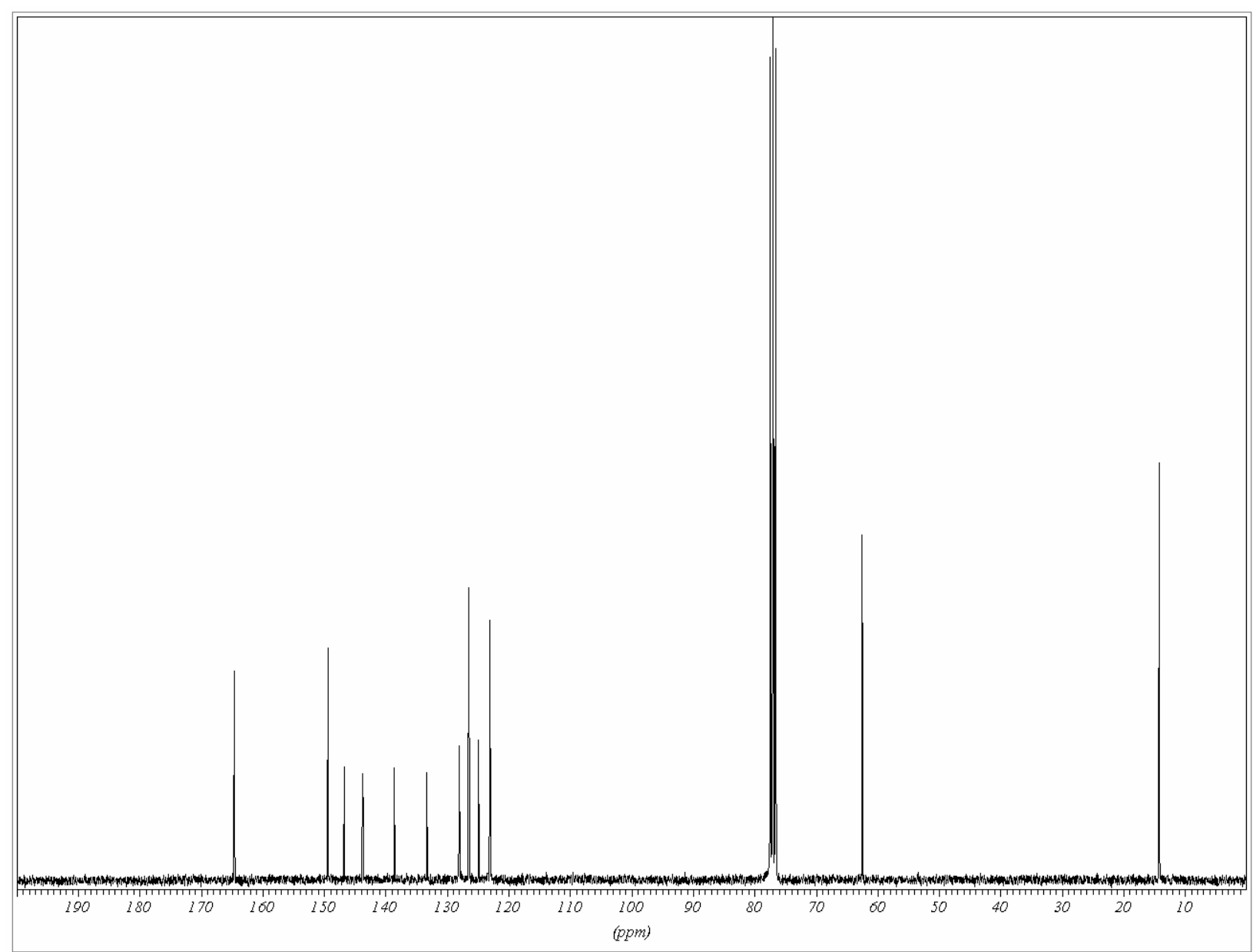

Figure S8. ${ }^{13} \mathrm{C}-\mathrm{NMR}$ of compound 2 in $\mathrm{CDCl}_{3}$. 


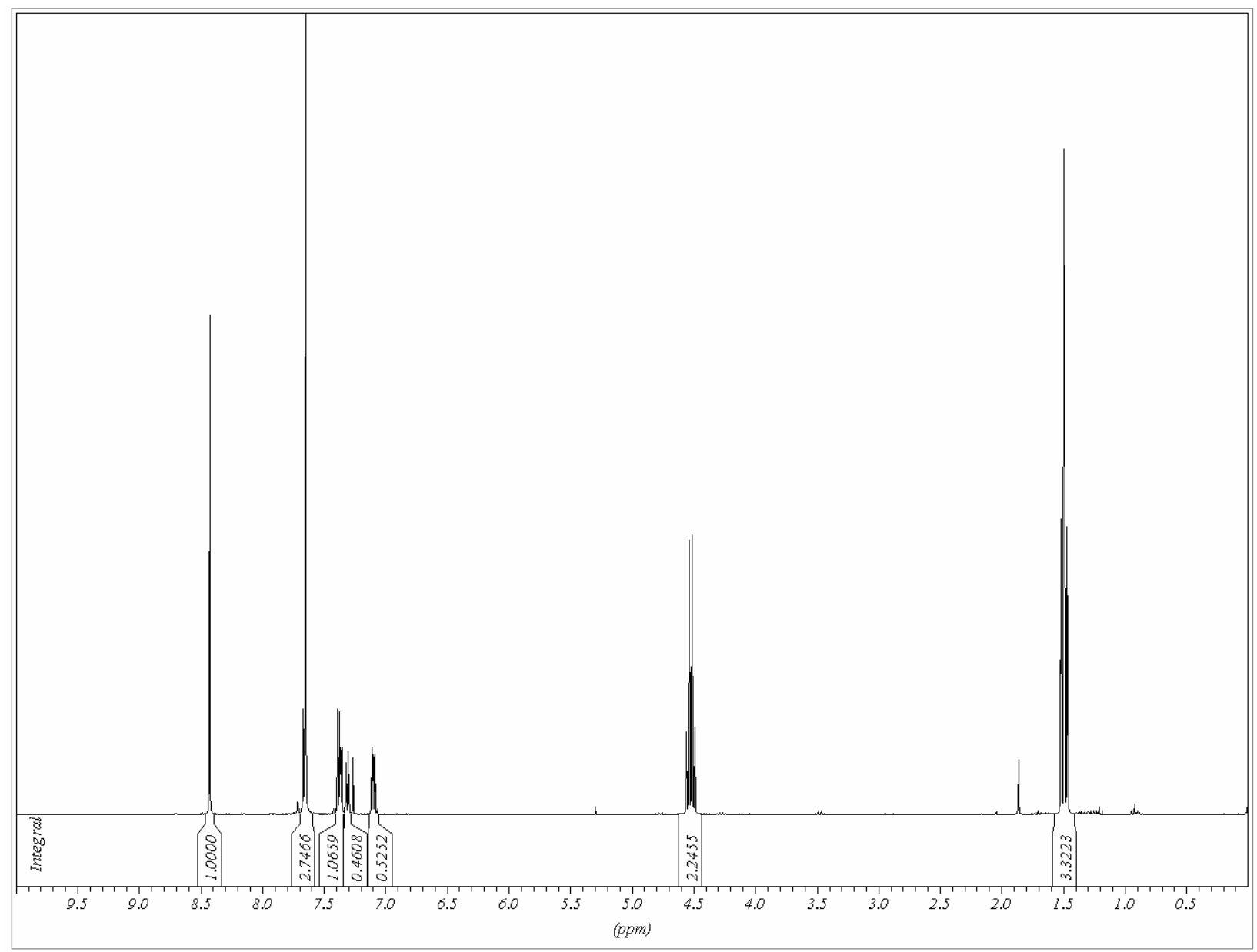

Figure S9. ${ }^{1} \mathrm{H}-\mathrm{NMR}$ of compound 3 in $\mathrm{CDCl}_{3}$. 


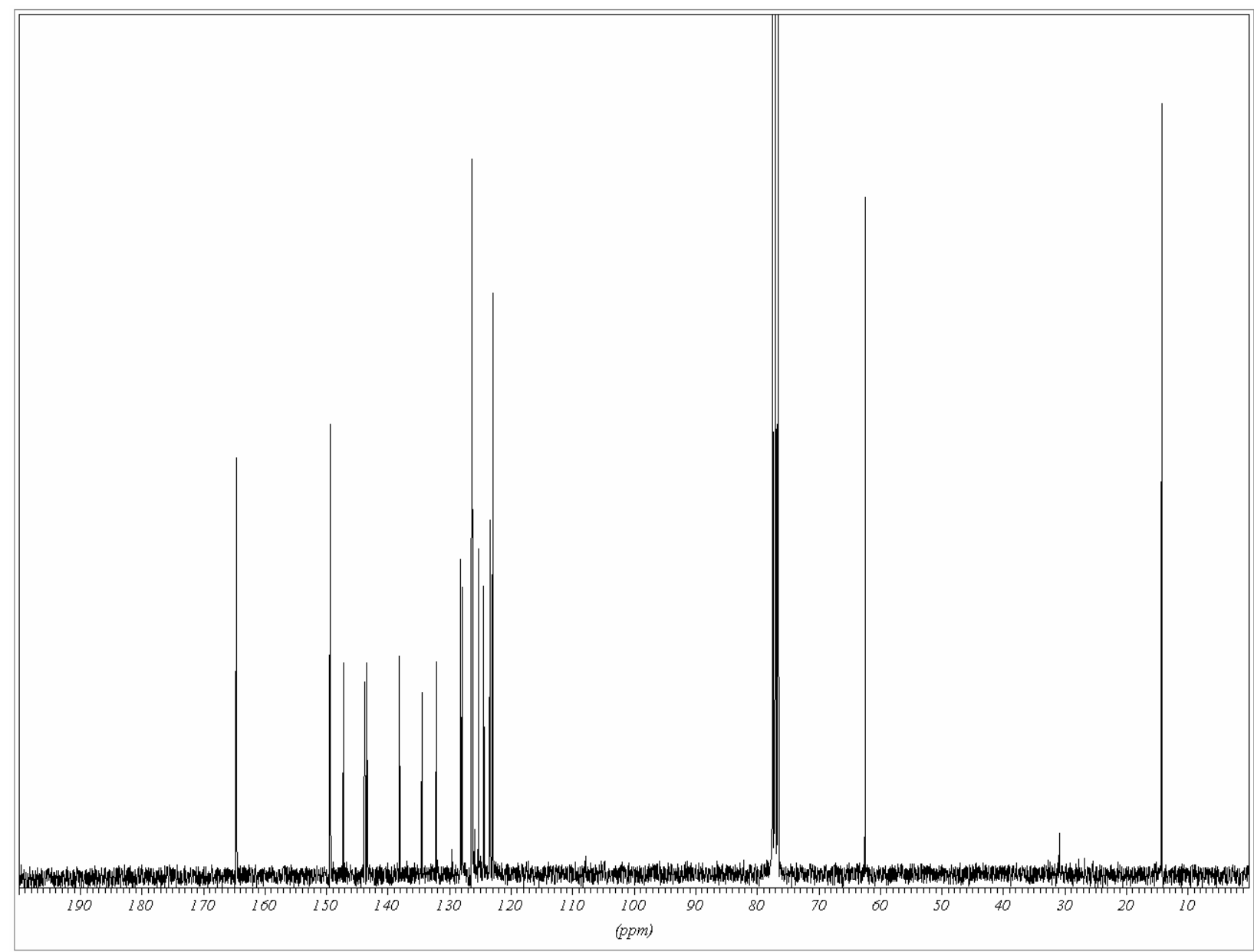

Figure S10. ${ }^{13} \mathrm{C}-\mathrm{NMR}$ of compound 3 in $\mathrm{CDCl}_{3}$.

\section{Bibliography}

(1) Zucchi, G.; Scopelliti, R.; Bünzli, J.-C. G., Journal of the Chemical Society, Dalton Transactions 2001, (13), 1975-1985.

(2) Wrighton, M. S.; Ginley, D. S.; Morse, D. L., Journal of Physical Chemistry 1974, 78, (22), 2229-33.

(3) Melhuish, W. H., Journal of the Optical Society of America 1964, 54, 183-6.

(4) Bril, A.; De Jager-Veenis, A. W., Journal of Research of the National Bureau of Standards, Section A: Physics and Chemistry 1976, 80A, (3), 401-7. 
(5) Hope, H., Prog. Inorg. Chem. 1994, 41, 1-19.

(6) SADABS: v.2.01, an empirical absorption correction program, Bruker AXS: Madison, WI, 2001.

(7) SMART: v.5.626, Bruker Molecular Analysis Research Tool, Bruker AXS: Madison, WI, 2002.

(8) SAINTPlus: v.6.36a, Data Reduction and Correction Program, Bruker AXS: Madison, WI, 2001.

(9) SHELXTL: v.6.10, Structure Determination Software Suite, Sheldrick, G.M., Bruker AXS: Madison, WI, 2001.

(10) M. J. Frisch, G. W. T., H. B. Schlegel, G. E. Scuseria, M. A. Robb, J. R. Cheeseman, J. A. Montgomery, Jr., T. Vreven, K. N. Kudin, J. C. Burant, J. M. Millam, S. S. Iyengar, J. Tomasi, V. Barone, B. Mennucci, M. Cossi, G. Scalmani, N. Rega, G. A. Petersson, H. Nakatsuji, M. Hada, M. Ehara, K. Toyota, R. Fukuda, J. Hasegawa, M. Ishida, T. Nakajima, Y. Honda, O. Kitao, H. Nakai, M. Klene, X. Li, J. E. Knox, H. P. Hratchian, J. B. Cross, C. Adamo, J. Jaramillo, R. Gomperts, R. E. Stratmann, O. Yazyev, A. J. Austin, R. Cammi, C. Pomelli, J. W. Ochterski, P. Y. Ayala, K. Morokuma, G. A. Voth, P. Salvador, J. J. Dannenberg, V. G. Zakrzewski, S. Dapprich, A. D. Daniels, M. C. Strain, O. Farkas, D. K. Malick, A. D. Rabuck, K. Raghavachari, J. B. Foresman, J. V. Ortiz, Q. Cui, A. G. Baboul, S. Clifford, J. Cioslowski, B. B. Stefanov, G. Liu, A. Liashenko, P. Piskorz, I. Komaromi, R. L. Martin, D. J. Fox, T. Keith, M. A. Al-Laham, C. Y. Peng, A. Nanayakkara, M. Challacombe, P. M. W. Gill, B. Johnson, W. Chen, M. W. Wong, C. Gonzalez, and J. A. Pople Gaussian 03 (Revision C.01), Gaussian, Inc.: Pittsburgh PA, 2003. 